BULLETIN OF THE

AMERICAN MATHEMATICAL SOCIETY

Volume 78, Number 4, July 1972

\title{
REMARKS ON SOME RESULTS OF GELFAND AND FUKS
}

\author{
BY VICTOR W. GUILLEMIN ${ }^{1}$
}

Communicated by I. M. Singer, December 17, 1971

By a topological Lie algebra over $\boldsymbol{R}$ we will mean a Lie algebra, $\mathscr{L}$, whose underlying vector space has a topology for which the bracket operation: $\mathscr{L} \times \mathscr{L} \rightarrow \mathscr{L}$ is continuous. One can associate with such a Lie algebra a complex, the $i$-cochains of which are all continuous alternating $i$-linear maps:

$$
\omega: \mathscr{L} \times \cdots \times \mathscr{L} \rightarrow \boldsymbol{R}
$$

and the coboundary operator defined by:

$$
d \omega\left(\zeta_{1}, \ldots, \zeta_{i+1}\right)=\sum(-1)^{j+k} \omega\left(\left[\zeta_{j}, \zeta_{k}\right], \zeta_{1}, \ldots, \zeta_{i}, \ldots, \hat{\zeta}_{j}, \ldots, \zeta_{i+1}\right)
$$

the cohomology of this complex will be denoted $H(\mathscr{L}, R)$. Gelfand and Fuks have proved the following remarkable result.

THEOREM. Let $X$ be a smooth compact oriented manifold. Let $\mathscr{L}$ be the Lie algebra of smooth vector fields on $X$ topologized by its $C^{\infty}$ topology. Then $H(\mathscr{L}, \boldsymbol{R})$ is finite dimensional in all dimensions.

See $[\mathbf{1}]$.

Figuring in their computations is a certain subcomplex of $(*)$ which they call the diagonal complex. It consists of all $i$-cochains $(*)$ having the property

$$
\omega\left(\zeta_{1}, \ldots, \zeta_{i}\right)=0 \quad \text { when } \operatorname{supp} \zeta_{1} \cap \ldots \cap \operatorname{supp} \zeta_{i}=\Phi .
$$

The cohomology of this diagonal complex they denote by $H_{\Delta}(\mathscr{L}, R)$. To describe their result about $H_{\Delta}(\mathscr{L}, \boldsymbol{R})$, consider the formal power series ring $\boldsymbol{R}\left[\left[x_{1}, \ldots, x_{n}\right]\right]$ generated by the $n$ indeterminates $x_{1}, \ldots, x_{n}$. The $\boldsymbol{R}$-linear derivations of this ring are a Lie algebra over $\boldsymbol{R}$ which we will denote by $L$. The $\mathscr{M}$-adic topology on the formal power series ring induces a topology on $L$. Let $H(L, R)$ be the cohomology of $L$ with respect to this topology. The result of Gelfand-Fuks is:

THEOREM. There is a spectral sequence whose $E^{2}$ term is the tensor product $H(X, R) \otimes H(L, R)$ and whose $E^{\infty}$ term is $H^{j}(X, R)$ for $j \leqq n$, and $H_{\Delta}^{j-n}(\mathscr{L}, R)$ for $j \geqq n$.

AMS 1970 subject classifications. Primary 22E65.

${ }^{1}$ Supported by NSF P22927. 
See [2].

Before stating our main theorem we will need to describe one other result of Gelfand-Fuks: Let $B_{u}$ be the universal classifying space for the group $U(n)$. Let $\left(B_{u}\right)_{2 n}$ be its $2 n$-skeleton. Let $P_{2 n}$ be the canonical principal $U(n)$ bundle setting above $\left(B_{u}\right)_{2 n}$.

THEOREM. $H(L, R) \cong H\left(P_{2 n}, R\right)$.

See Gelfand-Fuks [3].

This result suggests an obvious candidate for the spectral sequence described in the second theorem above. Namely, let $P_{X}$ be the principal $U(n)$ bundle associated with the complexified tangent bundle of $X$. $U(n)$ acts freely on the Cartesian product $P_{X} \times P_{2 n}$, and the quotient space $P_{X} \times P_{2 n} / U(n)$ is a fiber bundle over $X$ with fiber $P_{2 n}$. Therefore its Serre-Leray spectral sequence has the same $E^{2}$ term as the spectral sequence Gelfand and Fuks get for $H_{\Delta}(\mathscr{L}, \boldsymbol{R})$. Our main result is:

THEOREM. $H_{\Delta}^{i}(\mathscr{L}, R) \cong H^{i+n}\left(P_{X} \times P_{2 n} / U(n), R\right)$ for all $i \geqq 0$.

From this it is not hard to deduce:

COROLlary. The spectral sequence of Gelfand-Fuks is trivial if the Pontrjagin classes of $X$ vanish.

In [2] Gelfand and Fuks show that if the spectral sequence for $H_{\Delta}(\mathscr{L}, R)$ is trivial then a corresponding spectral sequence for $H(\mathscr{L}, R)$, whose $E^{2}$ term they know, is also trivial. This means one can compute $H(\mathscr{L}, R)$ for manifolds whose Pontrjagin classes are zero (for example spheres and compact Lie groups). Details will appear.

ADDEDIN PROOF. Gelfand and Fuks have informed me of some recent results of theirs which are very close to those described above.

\section{BIBLIOGRAPHY}

1. I. M. Gelfand and D. B. Fuks, Cohomologies of the Lie Algebra of tangent vector fields of a smooth manifold. I, Funkcional. Anal. i Priložen. 3 (1969), no. 3, 32-52. (Russian) MR 41 \# 1067.

2. - Cohomologies of the Lie algebra of tangent vector fields of a smooth manifold. II, Funkcional. Anal. i Priložen. 4 (1970), no. 2, 23-32. (Russian)

3. , Cohomology of the Lie algebra of formal vector fields, Izv. Akad. Nauk SSSR Ser. Mat. 34 (1970), 322-337 = Math. USSR Izv. 4 (1970), 327-342. MR 42 \#1103.

Department of Mathematics, Massachusetts Institute of Technology, Cambridge, MASSACHUSETTS 02139 